\title{
TYPE SYNTHESIS, MODELLING AND ANALYSIS OF THE MANIPULATOR FOR WHEEL-LEGGED ROBOT
}

\author{
JAROSŁAW SZREK*, ARTUR MURASZKOWSKI", PRZEMYSŁAW SPERZYŃSKI*
}

"Faculty of Mechanical Engineering, Department of Biomedical Engineering, Mechatronics and Theory of Mechanisms, Wroclaw University of Technology, Wybrzeże Wyspiańskiego 27, 50-370 Wrocław, Poland

jaroslaw.szrek@pwr.edu.pl, artur.muraszkowski@pwr.edu.pl, przemyslaw.sperzynski@pwr.edu.pl

received 11 December 2014, revised 14 March 2016, accepted 21 March 2016

\begin{abstract}
The aim of this article is to present the concept of wheel-legged mobile manipulator, which is a combination of mobile platform with specially selected suspension system and a manipulator. First, a literature review was performed and own solution proposed. The kinematic structure of manipulator, selected simulation results, physical model and the concept of the control system has been presented. Geometry synthesis was used to design basic dimension. Structural synthesis was performed according to the intermediate chain method. Simulations were performed using the multibody dynamics simulation software. New approach in the field of the mobile manipulators was presented as a result.
\end{abstract}

Key words: Mobile Manipulator, Multibody Dynamics Analysis, Wheel-Legged Platform

\section{INTRODUCTION}

A wheel-legged manipulator consists of mobile platform with special suspension system carrying a manipulator arm. Thanks to this solution the machine obtains additional functionality and offers new possibilities of application, such as remote manipulation in field with obstacles, exploration of unknown or contaminated areas (even other planets) and counter-terrorism task (Gronowicz et al., 2009; Szrek et al., 2010; Trojnacki et al., 2008).

There are numerous designs of mobile manipulators. The Inspector seen in Fig. 1a, Ibis (Fig. 1C), and PIAP Scout (Fig. 1d) were designed in the Industrial Research Institute for Automation and Measurements (PIAP, Poland) (http://www.antyterroryzm. com /product/pl/inspektor, http://www.antyterroryzm.com/product/ $\mathrm{pl} / \mathrm{scout}$ ). The robot Inspector has tracked chassis and manipulator with 5 degrees of freedom. Its main features are large weight and large payload of manipulator. The PIAP Scout platform uses different type of the drive system - wheel-track, which has better performance while overcoming obstacles.

The Ibis is a six-wheel platform equipped with a manipulator which has seven degrees of freedom and an impressive range of over 3 meters.

Each of the robots described above has a system of 4 cameras used for observation. Time of initialization from power off to standby is approx. $2 \mathrm{sec}$.

Another example of mobile robot is Seekur Jr (http://www. mobilerobots.com/Research Robots/SeekurJr_with_Outdoor_Man ipulator.aspx), presented in Fig. 2a. This platform has four nonswivel wheels and a manipulator with 5 degrees of freedom. An interesting feature is the use of a pneumatic actuator, as an additional support during the operation of the manipulator. Perpendicular to wheel motor placement suggests that worm gear has been used. Manipulator has approximately 1 meter range, weight $10 \mathrm{~kg}$, and maximum payload is dependent on the scope and varies from 3 to $15 \mathrm{~kg}$.
Another mobile manipulator is Herb (Srinivasa et al., 2009), shown in Fig. 2c. A class 2.0 mobile platform with additional support wheel is a moving module. The manipulator is equipped with an effector for picking various objects.

Interesting solution of manipulator (Fig. 2b) is used by GWAM robot designers (http://www.ams.com/eng). The manipulator has a modular construction and can be customized according to the needs. It can achieve up to seven degrees of freedom. This mobile platform moves on wheels, and additionally has track support, which is significant when driving in the rough terrain. The robot weights $139 \mathrm{~kg}$, and its payload is $9 \mathrm{~kg}$. The maximum speed of the robot is $3 \mathrm{~m} / \mathrm{s}$.

Another example is the KUKA robot (Fig. 2d). Mobile platform, which uses Mecanum wheels for omni-direction movement is equipped with a light industrial manipulator. Drive system used in this robot allows it to move mainly on hard and flat surfaces.

The main task for mobile manipulators is primarily to drive to a specific location and provide visual information to the operator. More complex task may consist of manipulation of the camera for more accurate analysis, provide additional sensory data or use of additional tools (eg. gripper, milling head). Further action may include grasping objects and loading on the platform.

To ensure that all the requirements are satisfied, the platform should have a chassis adapted to the task. If the action of the robot takes place on uneven terrain with obstacles task becomes much more difficult. A special case is when it is not possible to bypass obstacles and robot has to face it. In such a situation track drive system do well, especially equipped with an additional system lifting the front of the robot. However, the major disadvantage of solution is poor stability when moving over rough terrain. In this case, the wheel-legged suspension has the advantage.

Manipulators integrated with moving platform should have appropriate number of degrees of freedom to ensure correct manipulation during the task. Based on performed analysis, five degrees of freedom were assumed to be enough for manipulator performing most types of inspection tasks. 
a)

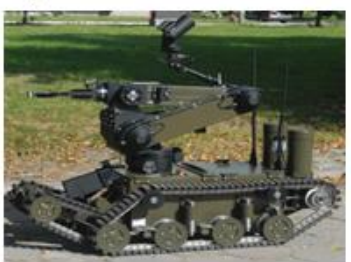

c)

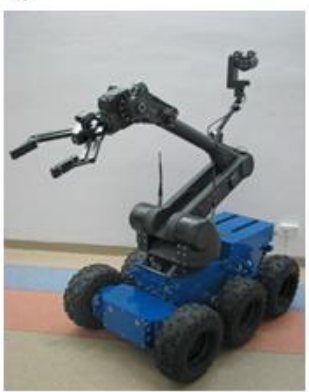

b)

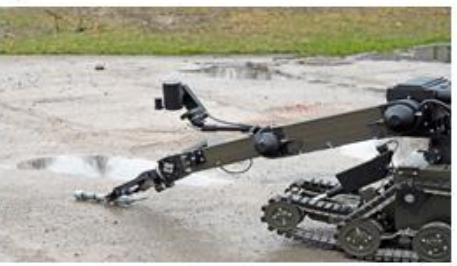

d)

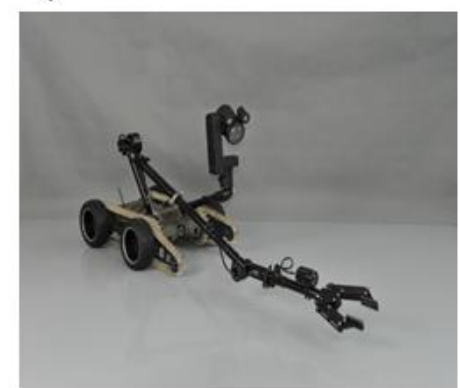

Fig. 1. Mobile robots produced by PIAP. a - Inspector manipulator when moving, $b$ - Inspector manipulator performing task, c-lbis, d-PIAP Scout

a)

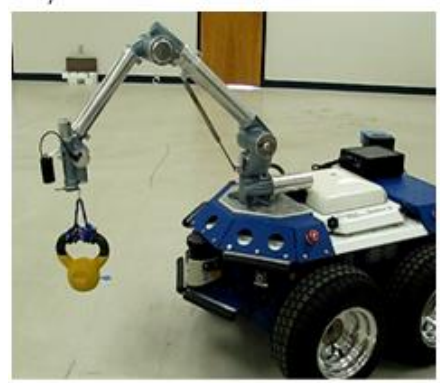

c)

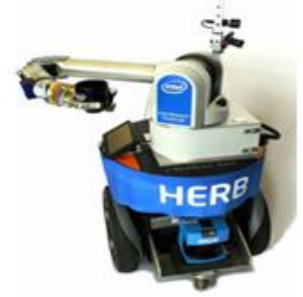

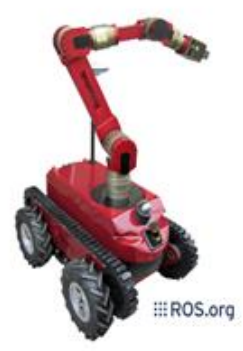

d)

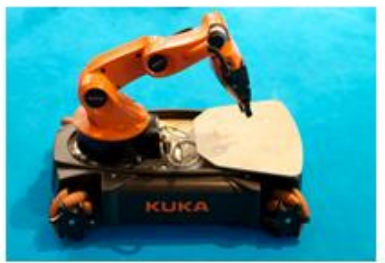

Fig. 2. Selected examples of mobile manipulators. a - Seekur Jr, b - Herb, c- GWAM, d - KUKA robot

\section{CONCEPT OF WHEEL-LEGGED MANIPULATOR}

Mobile manipulator designing started with identification of the overall assumptions and purposes. First, working area of the manipulator and its assumed location on the mobile platform - the wheel-legged robot (Bałchanowski et al., 2012a; Bałchanowski, 2012b; Gronowicz et al., 2009a; 2009b; 2012; Szrek et al., 2010), which view is shown in Fig. 3 , were defined.

Overall analysis of the construction of the manipulator due to the number of degrees of freedom and its structure was performed. In addition, types and location of joints were considered. The choice of the structure was performed using the method of intermediate chain (Gronowicz, 2003; Miller, 1988; Szrek, 2006) and the location of joints was dictated by practical considerations.

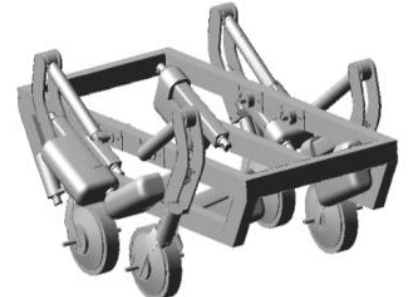

Fig. 3. Spatial model of mobile platform with wheel-legged suspension system

Next, the basic dimensions of elements were selected to ensure assumed range of movement and working area of manipulator. Each dimension was chosen for appropriate manipulator movements with regard to its mounting location on platform (the possibility of manipulation in front of the platform and below the mounting point).

\subsection{Kinematic Structure}

Kinematic scheme of manipulator is shown in Fig. 4a. It is assumed that the first joint of manipulator is placed in the geometric center of the platform. It will be a revolute joint $(A)$ with vertically oriented axis of rotation, which will turn the whole manipulator. Rest of manipulator's elements forms partially closed structure. A mechanism consisting of the elements 2, 3, 4, 5 has been selected due to the possibility of providing a determined trajectory (Buśkiewicz, 2014; Szrek, 2006; Sperzyński et al., 2010) with a single drive (the drive in joint $B$ ).

a)

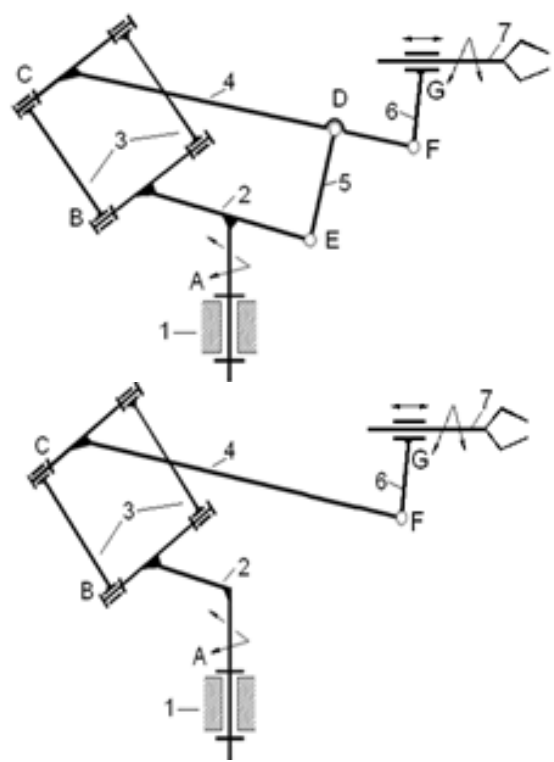

Fig. 4. Manipulator kinematic scheme: a) structure with four-bar linkage; b) serial structure

Similar principle is applied in the motion of the wheel-legged robot limbs, which resulted in reducing the number of drives needed for leveling function from eight to four (Gronowicz et al, 2009a). Moreover, in such a structure load is transferred via joints $B$ and $E$, and partially closed kinematic chain structure provide additional stiffness of the manipulator. 
The rest of the manipulator has a serial structure and consists of rotary joint $F$ and cylindrical joint $G$ (elongation and rotation of the effector).

During the selection process, the serial structure of the kinematics has been considered, shown in Fig. 4b. This solution simplifies the construction of the manipulator - less parts are needed, but they require one more drive.

\subsection{Computational model}

After selecting the structure and dimensions of the manipulator, model computing was performed. Figure $5 \mathrm{a}$ and $5 \mathrm{~b}$ shows the view of the manipulator along with examples of trajectories - straight and circular.

a)

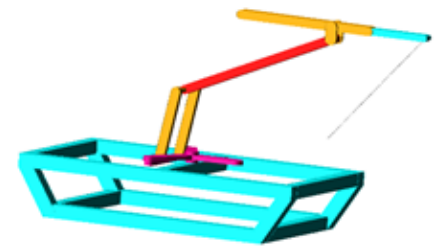

b)

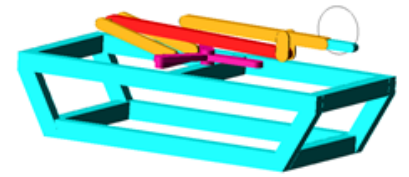

Fig. 5. Computational model of manipulator with platform: a) linear trajectory performance; b) circular trajectory performance

a)

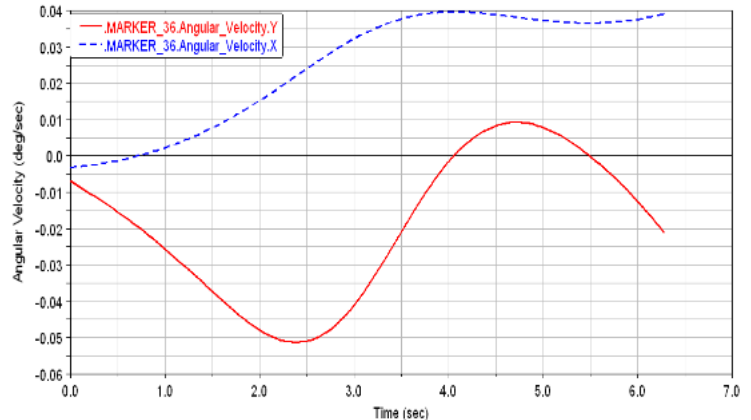

b)

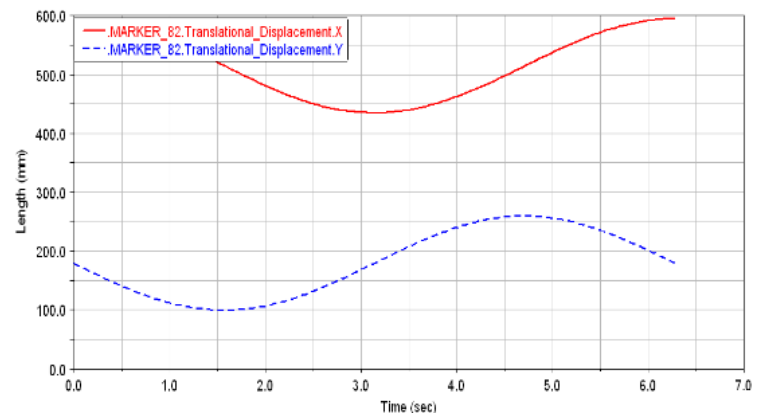

Fig. 6. Exemplary plots of circular trajectory simulation: angular velocity of element 3 ; b) displacement of element 7 ; component $x$ and $y$

In the next step, series of simulation tests was performed. Using appropriate software, both the kinematic and dynamic parameters were determined, such as range of motion, velocity, acceleration (linear, angular), forces and torque in kinematic joints and drives.

Exemplary plots are shown in Fig. 6. First plot (Fig. 6a) shows the components of the angular velocities of element 3 during executing circular trajectory. The waveforms of displacement along $x$ and $y$ axes of global coordinate system of element 7 is shown in Fig. $6 \mathrm{~b}$.

\subsection{Prototype}

After simulation tests, construction of the basic prototype of the manipulator was performed. Prototype was made according to previously determined kinematic structure and dimensions. The selected elements was made of steel and aluminium profiles. All revolute joints are mounted on bearings, and linear joint was made of two linear guideways with ball bearing arrangement. Fig. 7a shows the manipulator arm in the initial position where linear joint is arranged parallel to the arm 4 . Fig. $7 \mathrm{~b}$ shows the last element with the axis on which a gripper will be mounted.

a)

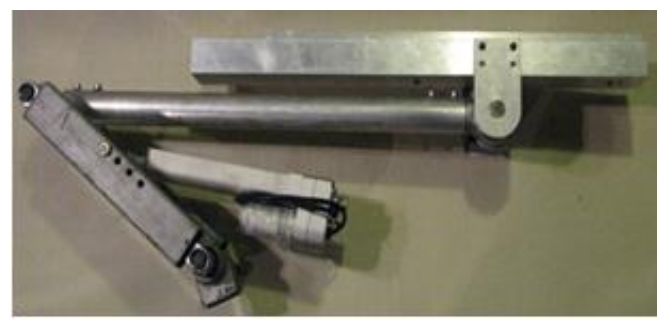

b)

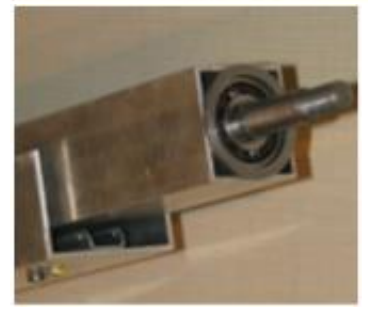

Fig. 7. Selected views of manipulator prototype: a) initial configuration view; b) linear and revolute joints of effector

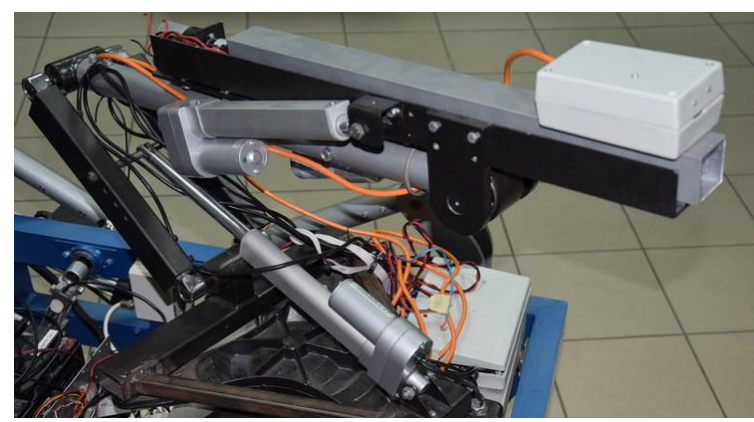

Fig. 8. Prototype of the manipulator on mobile wheel-legged platform

Manipulator was attached to wheel-legged mobile platform, shown in Fig. 10, and preliminary experimentally tested, where mechanical part correlations was studied.

\subsection{Control system}

The proposed manipulator has six degrees of freedom. The control system of the whole wheel-legged manipulator is responsible for the movement of the platform (drive module, leveling, stepping) and for the movement of the manipulator. Block diagram of the control system of the whole robot is shown in Fig. 8 with marked block responsible for the control of the manipulator. 
Due to the high complexity of the object, the control system is divided into functional blocks, and one of these blocks responsible for controlling the manipulator. Fig. 9 shows a more detailed structure diagram of the manipulator control system. Individual motors MOT 1-6 are driven by motor driver amplifiers (motor controllers) with PWM signal generated in the timers block located in modules with microcontroller MOD uC 1-3. The feedback loop is made with the quadrature signals from incremental encoders, which is mounted on each motor axle.

Motor controllers are determined as local modules in the structure - subordinate to the PC-Server placed on the platform.
Communication between the computer and the modules is carried out using the RS485 standard in accordance with communication protocol developed by authors.

Surveillance system is connected to two independent modules with higher performance required for image processing. Another reason to separate parts responsible for handling drive control from vision was to avoid interference. The software of the control system is based on the URBI package which has modules for task synchronization, which is necessary for controlling this type of device. The software is run and tested on a standard operating system, and ultimately will be set on the real-time system.

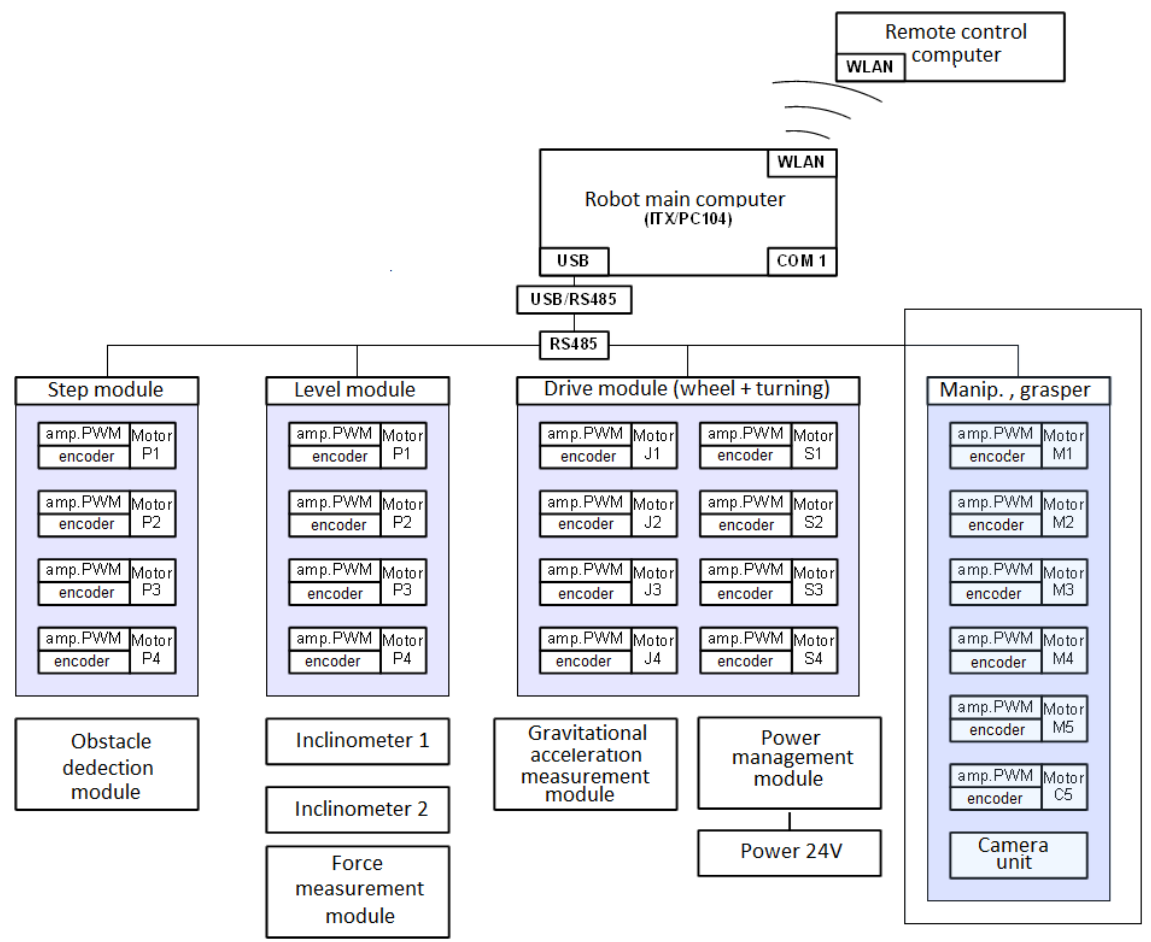

Fig. 9. Diagram of wheel-legged manipulator control system

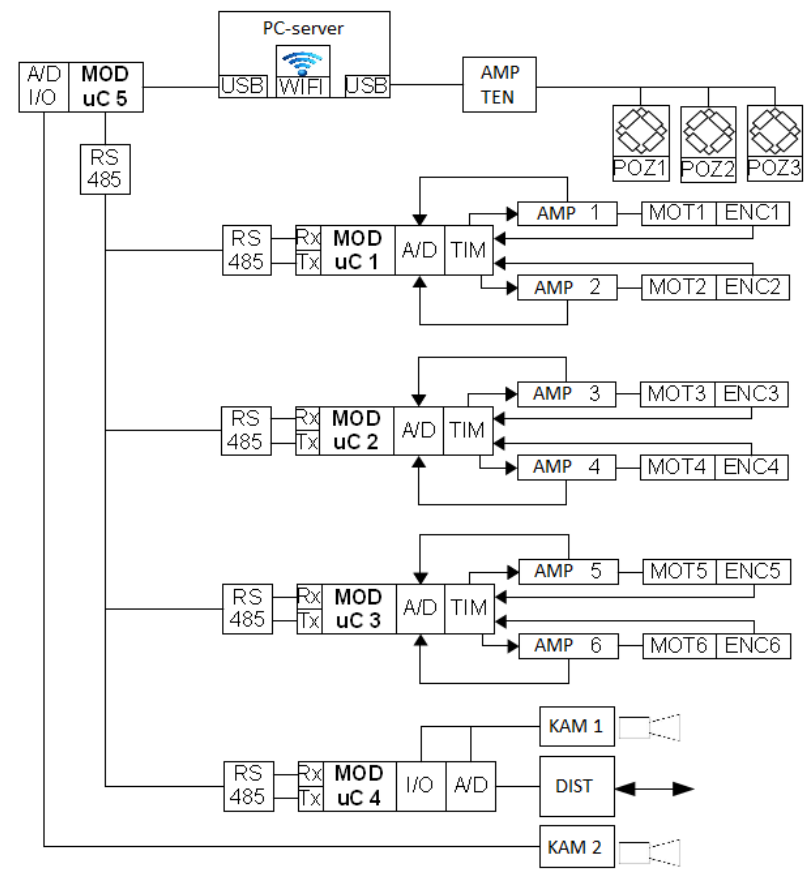

Fig. 10. Manipulator control system structure 


\section{SUMMARY}

Introduction to mobile manipulators and comparison of selected platforms was performed. Afterwards, own design was presented. Two kinematic structures shown in article were taken into account for later consideration. One of them was chosen and developed in prototype.

Design of four degree of freedom manipulator was presented. Construction of prototype of the manipulator was performed. Manipulator was preliminary tested for compatibility with wheellegged platform including work area, maximum range, dead zone and collisions. It has the ability to work witch different tools like graspers or electric tools, depending on the task. In the next step, it will be mounted on real wheel-legged platform and will become a base for the study of wheel-legged mobile manipulator.

Control system was also designed and presented in this paper. It has modular architecture for easy expansion and numerous comunication ports for attaching different sensors.

Furthermore, the idea of control system of wheel-legged mobile platform with attached manipulator was presented. Controlling such device is quite complex, due to the need of synchronization a large number of drives and cope in difficult robot environment uneven terrain with obstacles.

Designed control system has a modular structure, which allows adding new modules for both measurement and control of multiple DC electric motors.

Presented manipulator and wheel-legged platform integration is the new approach in the field of the mobile manipulators. The article proves, that this kind of integration is achievable. The main construction assumption of the manipulator, such as ability to reach objects from the surface on which the platform is moving, are sustained regardless of the platform kinematic configuration.

\section{REFERENCES}

1. Bałchanowski J. (2012b): Mobile wheel-legged robot: researching of suspension leveling system, Advances in mechanisms design / eds. Jaroslav Beran, Dordrecht, Springer.

2. Bałchanowski J., Gronowicz A. (2012a), Design and simulations of wheel-legged mobile robot, Acta Mechanica et Automatica, 6(2), 11-16.

3. Buśkiewicz J. (2014), Design of a feeder with the use of the path synthesis method - New Advances in Mechanisms, Transmissions and Applications Mechanisms and Machine Science, 17, 251-259.

4. Gronowicz A. (2003), Basis of kinematics systems analysis (in Polish), Oficyna wydawnicza PWr., Wrockaw.

5. Gronowicz A., J. Szrek (2009b), Design of LegVan wheel-legged robot's mechanical and control system, SYROM 2009 Red. Ion Visa, Springer Netherlands, 145-158.

6. Gronowicz A., Szrek J. (2009a), Idea of a quadruped wheel-legged robot, Archive of Mechanical Engineering, 56(3), 263-278.

7. Gronowicz A., Szrek J., Wudarczyk S. (2012), A rig for testing the leg of a wheel-legged robot, Acta Mechanica et Automatica, 6(2), 4852.

8. Miller S. (1988), Kinematic systems. Design basis (in Polish), WNT 1988, Warszawa.

9. Sperzyński P., Szrek J. Gronowicz A. (2010), Geometric synthesis of mechanism implementing linear trajectory (in Polish), Acta Mechanica et Automatica, 4(2), 124-129.

10. Srinivasa S. S., D. Ferguson, C. J. Helfrich, D. Berenson, Avaro Collet, R. Diankov, G. Gallagher, G. Hollinger, J. Kuffner, M. VandeWeghe (2009), HERB: a home exploring robotic butler, Auton Robot, 28, 5-20, Springer Science+Business Media.

11. Szrek J. (2006), Synthesis method of wheel-legged mobile robot suspension (in Polish), XX Konferencja Naukowo - Dydaktyczna Teorii Maszyn i Mechanizmów, Zielona Góra.

12. Szrek J., Wójtowicz P. (2010), Idea of wheel-legged robot and its control system design, Bulletin of the Polish Academy of Sciences. Technical Sciences, 58(1), 43-50.

13. Trojnacki M., Szynkarczyk P., Andrzejuk A. (2008): Trends of mobile land robots development (in Polish), Pomiary Automatyka Robotyka, 6.

14. http://www.ams.com/eng

15. http://www.antyterroryzm.com/product/pl/inspektor

16. http://www.antyterroryzm.com/product/pl/scout

17. http://www.mobilerobots.com/ResearchRobots/ SeekurJr_with_Outdoor_Manipulator.aspx

18. http://www.robotnik.es/en/contact/ 